\title{
¿EL FUTURO ES DE GENERADO? - APORTES PARA PENSAR EN UNA SOCIEDAD SIN ENCASILLAMIENTOS DE SEXO/GÉNERO
}

\section{¿WILL THE FUTURE BE GENDER-LESS? - KEY NOTES TO REFLECT ABOUT A SOCIETY WITHOUT SEX AND GENDER STEREOTYPES}

\author{
Victoria Grinstein \\ Casa de las Juventudes de la Ciudad Autónoma de Buenos Aires - Argentina \\ vicgrinstein@gmail.com
}

Resumen: Este artículo tiene como finalidad poner en discusión la categoría "sexo" presente en el documento nacional de identificación argentino. A partir de los aportes de la teoría queer y trans, se propone pensar los alcances posibles de modificar o eliminar esta categoría, en pos de crear una sociedad que se libere de los encasillamientos de género en términos registrales. Tomando distintas interpretaciones, se discute en torno a la construcción de la identidad a partir de la sanción de la Ley de Identidad de Género en el año 2012, sus alcances y limitaciones y las posibles implicancias sociales de pensarnos por fuera de la forma binaria que tenemos actualmente para categorizarnos. Para ello se utilizan los casos de varias personas travestis y no binaries en Argentina que lograron hacer cambios registrales para que en el casillero de "sexo" no aparezca ni hombre ni mujer.

Palabras clave: sexo, género, identidad, registro, encasillamientos.

\begin{abstract}
This article discusses about the category "sex" in the argentine identity card. Considering the queer and trans theory, it looks foward to the possibility of modifying this category to create a society free from gender clasifications. It considers different interpretations to argue about the construction of identity after the Gender Identity Law in Argentina was sanctioned en 2012, its goals and limitations and the possibility of imagining a society that breaks with binary categorizations. It uses a couple of examples from trans and non binary people in Argentina that managed to make changes in their ID, being able to register themselves without having to chose between female or male.
\end{abstract}

Key words: sex, gender, identity, registry, stereotypes. 


\title{
1. Introducción
}

\author{
degenerado, degenerada \\ 2. adjetivo $\cdot$ nombre masculino y femenino \\ [persona] Que tiene una conducta sexual que se considera fuera de lo normal o de lo moralmente \\ aceptado. \\ "me llaman criminal, me tachan de loco y de degenerado"l
}

Algunos meses me volví a encontrar con una antigua cédula de identidad que me llamó profundamente la atención. Al lado de una fotografía de algún antepasado, había un largo listado de características que le atribuían a su persona: estatura, color, ojos, pelo, señas naturales y defectos físicos. La lista seguía y recién en la segunda página aparecía la categoría sexo, la única sobreviviente del modo de categorizar a las personas de principio del siglo XX.

La identidad registral de las personas de aquel entonces se construía a partir de este sinfín de atributos físicos en consonancia con el modelo positivista científico de la época, el cual se hermanaba con el Estado para la construcción de sujetxs. Estaba completamente naturalizada la categorización de las personas a partir de ciertas características corporales, con la cual hoy probablemente nos horrorizaríamos. La interpretación social de las personas a partir de ciertos rasgos fenotípicos se encuentra incluso penalizada y sería imposible sostenerse en los marcos regulatorios de derechos humanos.

Pero casi 100 años después, nos seguimos encontrando con documentos de identidad que siguen categorizando y encasillando a las personas de forma muy limitada. Quizás ya no tenga que ver con el tamaño de la nariz o el color de piel, pero ciertas características corporales siguen siendo las que nos determinan de por vida. Hembra/Macho, Mujer/Varón, son las únicas posibilidades que tenemos y que determinaran nuestra trayectoria vital. Este articulo tiene como finalidad preguntarse, ¿Si logramos problematizar categorías racistas y discriminadoras defendiendo la integridad de las personas, lograremos algún día superar la categoría de sexo y construirnos por fuera de esta? ¿Es posible romper con clasificaciones genéricas?

Históricamente la sociedad estuvo dividida en dos: hombres por un lado, mujeres por el otro. Este modelo binario y determinista es el que ha impregnado las teorías científicas que llegan hasta el siglo XXI y que siguen pensando a los cuerpos de forma estática en una escala que va de macho a hembra como posibilidades de lo normal. La sociobiología y el darwinismo social contribuyeron a cimentar la idea de que la naturaleza biológica de los cuerpos determina el lugar que cada persona ocupa dentro de la sociedad y por ende la jerarquía superior de los hombres blancos por sobre el resto (Maffia, 2004).

\footnotetext{
${ }^{1}$ Definición de Oxford Languages, Recuperado de https://languages.oup.com/google-dictionary-es/. IQUAL. REVISTA DE GÉNERO E IGUALDAD, 2022, 5, 116-126 
Como afirma Fausto-Sterling (1998), este modelo binario marca no solamente un modo de ser hombre-mujer sino también un modo de desear heteronormativo que encaje con los ideales de reproducción vigentes en todas las teorías científicas. El discurso neurocientífico se instaura como el nuevo paradigma legitimador de las diferencias entre hombres y mujeres. Con un listado más amplio de órganos para analizar que siglos anteriores, pero con la misma idea reduccionista sobre los cuerpos. La comunidad médica se encarga de eliminar cualquier tipo de corporalidad que no encaje dentro de los parámetros heterosexuales de macho y hembra.

Va a ser desde los feminismos y con la incorporación de las mujeres a esferas del pensamiento, donde todas estas ideas impregnadas de sexismo e injusticia epistémica comiencen a resquebrajarse. A pesar de una gran resistencia de las comunidades científicas, surgen discursos que disputan estas concepciones que continúan siendo androcéntricas y sumamente excluyentes

Según Butler (1995) los cuerpos surgen y perduran dentro de las limitaciones productivas de ciertos esquemas reguladores generizados. Estos encasillamientos funcionan como una restricción constitutiva que produce un terreno de cuerpos inteligibles, así como también un terreno de cuerpos invivibles e impensables. En este sentido podemos pensar la existencia de la categoría "sexo" en el documento de identidad como un esquema regulador de cuerpos normalizados necesario para el ordenamiento social. Para muchas personas sería impensable una sociedad que no esté generizada, dividida y clasificada.

De todos modos, Butler (1995) hace un llamamiento a desestabilizar este orden obligatorio que encadena sexo, género y deseo en una matriz heterosexual. Centrándose en el concepto de performatividad, va a pensar al género como un conjunto de haceres y significados establecidos socialmente, que puede estar sujeto a ser modificado a lo largo del tiempo.

Preciado (2002) también adscribe a esta posibilidad de transformación, poniéndole fin a un sistema que diferencia entre hombres y mujeres, para sustituirlo por uno que entienda a las personas por fuera de una identidad sexual cerrada y esencialista. La sociedad contrasexual se niega al disciplinamiento heteronormativo de los cuerpos para sustituirlo por una producción de formas de placer-saber alternativas a la sexualidad moderna.

En términos foucaultianos, el sexo no sólo funciona como norma, sino que además es parte de una práctica reguladora que produce los cuerpos que gobierna. Se impone como una fuerza reguladora que se manifiesta como poder productivo, el poder de producir, circunscribir y diferenciar los cuerpos que controla (Foucault, 1976). Cabe preguntarnos, si es posible pensar una sociedad que construya y produzca por fuera de esta categorización. $Y$ si superamos este modo de ordenamiento en pos de un mundo des-generizado, ¿qué nuevas categorías surgirán para permitir nombrarnos? 


\section{Críticas a la Ley de Identidad de Género}

La Ley de identidad de género se sancionó en Argentina en 2012, y marcó un antes y un después para la comunidad trans en el país. Pionera en materia de derechos en América Latina, esta ley vino a discutir el modelo cis genérico de identificación de las personas y a permitir traducir las militancias de las personas trans en políticas públicas.

Esta Ley es sumamente innovadora, ya que a diferencia de otros países que requieren de procedimientos de vigilancia corporal, reafirma el carácter de las personas de decidir sobre su propia identidad de género sin tener que pasar por controles médicos o psicológicos (Rueda, 2019). En el art. 2 se establece que

se entiende por identidad de género a la vivencia interna e individual del género tal como cada persona la siente, la cual puede corresponder o no con el sexo asignado al momento del nacimiento, incluyendo la vivencia personal del cuerpo. Esto puede involucrar la modificación de la apariencia o la función corporal a través de medios farmacológicos, quirúrgicos de otra índole, siempre que ello sea libremente escogido. (p. 7)

De este modo se abre un nuevo paradigma para pensar identidades y corporalidades en tanto deja de existir un único modelo para vivir una masculinidad o una feminidad. La ley posibilitó que muchxs puedan transitar sus vidas sin estar condenadxs por la genitalidad, desafiando fuertemente los mandatos patriarcales.

De todos modos, desde la comunidad travesti, incluso luego de haber militado y defendido esta ley, se han realizado ciertas criticas por la continuidad de su carácter binario y la imposibilidad de reconocimiento de la identidad travesti. Marlene Wayar (2012), histórica militante trans, festejaba la promulgación de la ley, al mismo tiempo que se preguntaba "¿Qué pasó con la T?", haciendo referencia a la limitación del cambio registral, el cual solo permite moverse dentro de las categorías Hombre y Mujer.

De este modo, el Estado no logra leer ni visibilizar aquellas identidades que se perciben por fuera de este binario. Por el contrario, continúa normalizando los cuerpos en un sistema limitante que solo admite dos categorías posibles (De Mauro Rucovsky, 2019). La potencia disruptiva de la ley frente a una sociedad heterocispatriarcal se ve limitada ya que aquellas personas travestis como es el caso de Wayar, o como del colectivo no binarie, continúan quedando por fuera de la inscripción y el reconocimiento estatal.

Existen varios casos emblemáticos para repensar los alcances de la ley de identidad de género. La activista travesti Lara Bertolini presentó el año pasado un recurso extraordinario ante la Cámara Nacional de Apelaciones en lo Civil con el 
objetivo de que se consigne tanto en el DNI como en la partida de nacimiento su identidad: 'femineidad travesti'. Ella afirmaba:

En la actualidad no permitir el pleno reconocimiento de las identidades no binarias, en específico las identidades travestis y transgéneros, implica una condena a vivir en un inframundo social, alejadas de toda posibilidad de acceso a derechos básicos. Esta situación implica desconocer que el principio de la dignidad humana se basa en el reconocimiento de las identidades en toda su multiplicidad ${ }^{2}$.

En marzo del año pasado, había logrado un fallo inédito que establecía en el campo asignado para sexo de su DNI la expresión femineidad travesti. Esta posibilidad de nombrarse de acuerdo a su identidad de género no solo implicaba un avance personal, sino que ponía en discusión siglos de binarismo y encasillamientos de género. Se abría la posibilidad de pensarnos e identificarnos con mucha mayor libertad que lo hasta ahora conocido. De todos modos, esta decisión fue apelada por el Registro Nacional de las Personas (RENAPER) que se negó a abrir un nuevo campo bajo el argumento de que "solo hay dos sexos".

Mientras tanto los casos de personas no binaries que desean hacer cambios en sus documentos de identidad se multiplican. En 2018 se dio la primera modificación inédita en el mundo de un DNI que no indicaba el sexo de la persona. En la provincia de Mendoza, el Registro Civil se hizo eco del pedido de dos personas que no se identificaban con la categoría femenino y masculino, permitiéndoles escribir "ninguno" en el casillero consignado para el sexo. La abogada Eleonora Lamm, quien llevó adelante este caso se pronunciaba:

Nuestra ley de identidad de género dice que tu identidad es cómo vos te sentís, independientemente de la biología. ¿Para qué se sigue consignando un sexo en el DNI? Es una incoherencia legal; a la ley no le interesa la biología. No es reflejo de la identidad (Vallejos, 2018).

Mauro Cabral (2014), militante intersex se suma a la discusión también afirmando:

La ley de identidad de género argentina ha sido y es frecuentemente criticada en espacios autopercibidos como radicales porque limita el reconocimiento legal a dos sexos -lo que es decir, limita el cambio registral del sexo de femenino a masculino o viceversa, sin admitir la inscripción de un sexo distinto a femenino o masculino-. Esta es, sin dudas, una cuestión sobre la que será necesario regresar -a través de la exploración de las potencialidades del proceso administrativo, a través de litigio estratégico, o de futuras revisiones legislativas de la ley, de su reglamentación y de su implementación-. Es posible, sin embargo, que la superación futura de la Ley de

\footnotetext{
2 Apelan ante la Justicia el no reconocimiento de femineidad travesti en DNI (26/12/2019). Agencia Presentes. Recuperado de https://agenciapresentes.org/2019/12/26/apelan-ante-la-justicia-el-noreconocimiento-de-femineidad-travesti-en-dni/
} 
Identidad de Género argentina no necesariamente implique el reconocimiento de más sexos o de más géneros -sino, y lo digo con esperanzas, la abolición de ambas nociones como categorías jurídicamente relevantes. (p. 212)

Estas disputas en el campo de lo legal nos remiten a las discusiones generadas por distintxs teoricxs queer y sus aportes para pensar una sociedad más libre e inclusiva. El Manifiesto Contrasexual de Preciado alude a la pregunta que se hace Lamm respecto a la relación entre identidad y biología. Para él, la contrasexualidad aparece como el fin del ordenamiento de los cuerpos a partir de la Naturaleza. En este sentido, los cuerpos dejan de ser reconocidos como hombres o mujeres y son pensados tan solo como cuerpos hablantes, que reconocen a Ixs otrxs como cuerpos hablantes. Se produce una renuncia a una identidad sexual cerrada que deconstruye el sistema de naturalización del género. La sociedad contrasexual busca la equivalencia de todos los cuerpos-sujetxs hablantes dedicadxs a la búsqueda del placer-saber rompiendo las divisiones dicotómicas con las cuales todavía nos construimos (Preciado, 2000).

A fines de 2019, volvía a salir en el diario el caso de una persona que también recurría a la Justicia para modificar su documento de identificación. En Tierra del Fuego, tras varios meses de lucha, el Registro Civil de Ushuaia autorizaba a que en el documento de la persona aparezca el rótulo 'no binario'. Este representa el primer fallo judicial de esta clase en la historia de la provincia y uno de los primeros en el país. ${ }^{3}$ Esta medida nuevamente pone en duda la legislación actual y los alcances de la Ley de Identidad de Género que no reconoce la multiplicidad de subjetividades que cada vez logran mayor notoriedad en nuestra sociedad. Del mismo modo que nos hemos apropiado de los insultos "puto"; "marica"; "torta" y "trava", quizás sea hora también de reclamar el reconocimiento de estas identidades por parte del Estado.

La propuesta de revisar la categoría "sexo" de los documentos de identidad alude a una nueva forma de pensar la subjetivación y la producción corporal en términos menos restrictivos que permitan la incorporación de otros marcos de reconocimiento de las personas. Berkins (2003) también defendía la erradicación de los encasillamientos en identidades preconstruidas por un sistema opresor. Para una de las más importantes activistas travesti de nuestro país la deconstrucción de las dicotomías jerarquizadas que se nos imponen debe ser nuestra meta. El travestismo implica un no identitarismo. Una ruptura con un modo de entender las identidades en términos limitantes, separatistas y excluyentes.

\footnotetext{
3 Redacción Barcelona (18/12/2019) "Autorizan a una persona a llevar en su DNI el género no binario". España. Recuperado dehttps://www.lavanguardia.com/vida/20191218/472325197671/argentina-dnigenero-no-binario-hombre-mujer-registro-civil.html IQUAL. REVISTA DE GÉNERO E IGUALDAD, 2022, 5, 116-126 ISSN. 2603-851X DOI. http://dx.doi.org/10.6018/iqual.467071
} 


\section{3. ¿El futuro es de generado?}

Preciado (2000) nos ofrece pensar una sociedad con una producción de formas de placer-saber alternativas a la sexualidad moderna. Las prácticas contrasexuales se oponen al disciplinamiento sexual que nos ofrece como única posibilidad la construcción de identidades a partir de las oposiciones hombre/mujer, masculino/femenino, heterosexualidad/homosexualidad. La creación de estas diferencias sexuales, supuestamente amparadas en características naturales, no es más que una operación tecnológica de reducción que analiza determinadas partes de la totalidad del cuerpo y las aísla para hacer de ellas significantes sexuales. Así como antes estábamos segregadxs por nuestro color de piel, hoy seguimos estando separadxs por el sexo al cual nos asignaron o en algunos países al menos según el que elegimos.

Por ende, la separación y categorización de cuerpos en hombres y mujeres, forma parte de una construcción dentro de un sistema heterosexual de producción y de reproducción que autoriza el sometimiento de ciertos cuerpos por sobre otros. Las consecuencias sobre aquellos cuerpos que se consideran inferiores con devastadoras. Las cifras de femicidios y travesticidios siguen siendo alarmantes, mientras que no existe oportunidades para quienes deciden salirse del mandato social. En este sentido, resulta necesario pensar en la construcción nuevas alternativas para la construcción identitaria. Remitiéndonos a Preciado podemos pensar en la edificación de una contrasexualidad que sacuda las tecnologías de la escritura del sexo y del género, así como sus instituciones (Preciado, 2000).

Silva Horne, diputada rionegrina, con el apoyo de la FALGBT presentó hace algunos años un proyecto de ley contra la asignación sexual compulsiva, el cual buscaba la eliminación de la categoría sexo en documentos en pos de la protección de la diversidad corporal. Como se describe en el proyecto, la finalidad de la ley es poder garantizar el derecho a la intimidad, la autonomía, la autodeterminación, la integridad y la diversidad corporal de todas las personas, en especial de aquellas vulneradas por la discriminación bajo el pretexto de que sus cuerpos no hegemónicos varían de normas construidas sobre la base de una falsa noción de sexo. Incorporando la lucha intersex, aspira a proteger a las personas de posibles intervenciones (o mutilaciones) destinadas a modificar sus características sexuales para adecuarlas a los estándares médicos de asignación sexual o "normalidad" (Cabral, 2018).

Siguiendo los postulados de Butler (1995) podemos pensar la categoría del sexo en términos normativos y discursivos, más que aludiendo a su materialidad. Son los discursos y las normas los que dictan el modo en que nos identificamos. Por ende, la propuesta es sumamente transformadora ya que al eliminar la categoría de "sexo" de cualquier documento público o privado, se asume la irrelevancia jurídica de los datos sobre la genitalidad de las personas. A su vez, la prohibición de tratamientos médicos 
irreversibles e intrusivos sobre las características sexuales de una persona, se opone a la "normalización" y estandarización de los cuerpos.

Probablemente para Butler (1995) no tendría sentido ampliar las categorías de sexo/género y sumarlas al binomio hombre-mujer, ya que de este modo se seguirían generando nuevos ámbitos de exclusión. Podemos pensar que, según su análisis, la eliminación de cualquier categorización resultaría también en la eliminación de su fuerza disciplinadora. En este sentido, una desidentificación colectiva ayudaría a alcanzar una reconceptualización de aquellos cuerpos que importan y aquellos que son desechables para nuestra sociedad, es decir que serían más aquellxs que podrían gozar de una "vida vivible".

Quizás pensar hoy en un proyecto como el presentado por Horne nos suene un tanto utópico. Incluso hoy, contando con la Ley de Identidad de Género, la organización de los colectivos travesti, trans, no binaries e intersexuales, se hace indispensable para defender los derechos todavía vulnerados de muchas personas. Si aún hoy seguimos padeciendo la violencia institucional en ámbitos de salud, laborales y educativos, parecería difícil lograr la aceptación de un proyecto revolucione la construcción de la identidad como el de la diputada.

De todos modos, es siempre valioso recuperar los aportes de Muñoz en torno a la utopía queer. El proyecto utópico queer se distancia del esfuerzo apagado de la homosexualidad que desea la normalidad y lo práctico. Va más allá del presente asimilacionista, buscando forjar una política con nuevas formas de nombrar la vida y de vivir esos nombres (Muñoz, 2009). Quizás sea esta Utopía Queer la que guíe la búsqueda de una acción política que no implique una mera inclusión a un orden social que se ha jactado de sus efectos discriminatorios y estigmatizantes, sino que suponga otro modo de existir en el mundo y de construir comunidad.

\section{Conclusiones}

Los cuerpos que no encajan dentro de los patrones normalizadores de la sociedad han sido históricamente segregados y marginados, privados de derechos y de calidad de vida. Excluidos de ámbitos laborales, educativos, sociales y familiares, han luchado por sobrevivir y abrirse paso ante un mundo que niega su existencia. Es por ello que los movimientos disidentes que han crecido y se han multiplicado tanto en la Argentina como en el resto del mundo merecen un enorme reconocimiento. La conquista de la Ley de Identidad de Género fue un enorme paso en cuanto a la ampliación de derechos y una demostración política para los grupos conservadores de derecha que se oponían a su sanción. Argentina se volvió un ejemplo para los activismos de los países de la región que aun pelean por ser reconocidxs ante la ley.

Acompañando estas luchas, desde la teoría queer como desde la teoría trans se viene problematizando la categoría de sexo/género y el modo en que opera en nuestra IQUAL. REVISTA DE GÉNERO E IGUALDAD, 2022, 5, 116-126 ISSN. 2603-851X DOI. http://dx.doi.org/10.6018/iqual.467071 
sociedad. Considerando sus efectos productivos, al igual que su impacto violento sobre los cuerpos, se han buscado distintas salidas que rompan con la operación normalizadora que pretende imponer el sistema sexo genérico.

Una de estas se propone cuestionar su construcción en términos jurídicos y registrales, específicamente en lo que respecta a la categoría "sexo" en el documento de identificación de las personas. Cuestionando el carácter binario de la Ley de Identidad de Género actual, se proponen distintas salidas que rompan esta categorización dicotómica, ya sea incorporando nuevas identidades como directamente aboliendo esta categoría en documentos registrales.

De todos modos, cabe preguntarnos si la reproducción del binarismo tiene que ver estrictamente con esta ley o si forma parte de un andamiaje más dentro de un dispositivo heterocisnormativo y disciplinador que determina nuestras formas de vida. Estar inmersxs en un sistema opresor, binarista y heterocisexual nos impide pensar en salidas que permitan desmantelar estos mecanismos. Cabe preguntarnos si la ley de identidad de género forma parte de las distintas estrategias de ruptura o sigue siendo parte del problema.

A pesar de que la ley no subordina el reconocimiento legal de la identidad a la exploración psicofísica de quienes solicitan un cambio registral ni requiere la portación del "cuerpo correcto", sigue imposibilitando una definición por fuera de hombre/mujer. Por eso nos vemos ante la necesidad de seguir formulando nuevas respuestas que incluyan a quienes continúan quedando excluidxs. Al día de hoy, incluso contando con una ley que refiere a la identidad de género autopercibida, siguen habiendo personas que no logran ser nombradas, que deben adscribir a un sexo/género al cual no se sienten parte y por ende que pierden parte de su libertad subjetiva.

La discusión en torno a estos proyectos continúa abierta y sujeta al desarrollo de nuevas posturas que cuestionan el modo en que nos construimos identitariamente. Quizás pueda parecer una discusión menor mientras las cifras de travesticidios y los crímenes de odio se mantienen en alza, o cuando aún no hay un proyecto de ley que contemple la inclusión laboral de personas trans. De todos modos, se vuelve indispensable pensar en los posibles alcances de una política que trastoque las categorías hombre/mujer y que admita la existencia de múltiples identidades. Nos permitimos preguntarnos si efectivamente, ¿el futuro es de generado?

\section{Bibliografía}

Apelan ante la Justicia el no reconocimiento de femineidad travesti en DNI. (26 de diciembre de 2019). Agencia Presentes. Recuperado de https://agenciapresentes.org/2019/12/26/apelan-ante-la-justicia-el-noreconocimiento-de-femineidad-travesti-en-dni/ 
Autorizan a una persona a llevar en su DNI el género no binario. (18 de diciembre de 2019). La Vanguardia. Recuperado de https://www.lavanguardia.com/vida/20191218/472325197671/argentina-dnigenero-no-binario-hombre-mujer-registro-civil.html

Berkins, L. (2009). Un itinerario político del travestismo. En D. Maffía (comp.) Sexualidades migrantes. Género y transgénero, Buenos Aires, Argentina: Feminaria y Librería de Mujeres.

Butler, J. (2015). El género en llamas: cuestiones de apropiación y subversión", Cuerpos que importan. Sobre los límites materiales y discursivos del sexo. Buenos Aires, Argentina: Paidós.

Cabral, J. (15 de noviembre de 2018). DNI sin sexo: "Es un proyecto a favor de la comunidad más vulnerada de la sociedad". Tiempo Argentino. Recuperado de https://www.tiempoar.com.ar/nota/dni-sin-sexo-es-un-proyecto-a-favor-de-lacomunidad-mas-vulnerada-de-la-sociedad.

Cabral, M. (2014). Derecho a la igualdad: Tercera posición en materia de género. Corte Suprema, Australia, NSW Registrar of Births, Deaths and Marriages v. Norrie, 2 de abril de 2014. Revista Derechos Humanos, 3(8), 199-212.

De Mauro Rucovsky, M., \& Russell, I. (2019). The Travesti Critique of the Gender Identity Law in Argentina. Transgender Studies Quarterly, 6(2), 223-238.

Fausto-Sterling, A. (1998). Los cinco sexos. Transexualidad, transgenerismo y cultura: Antropología, identidad y género, 79-90.

Foucault, Michel, (1998). El dispositivo de la sexualidad, Historia de la sexualidad 1: la voluntad del saber, Ciudad de México, México: Siglo Veintiuno.

Maffía, D. (2007). Epistemología feminista: la subversión semiótica de las mujeres en la ciencia. Revista Venezolana de Estudios de la Mujer, 12,(28).

Ministro de Justicia y Derechos Humanos. (2012) Ley $n^{\circ} 26.743$, Identidad de Género. Recuperado de: www.jus.gob.ar/media/3108867/ley_26743_identidad_de_genero.pdf

Muñoz, J. E. (2020). Lo queer como horizonte. Hermenéutica de la Utopía frente al pragmatismo gay. Utopía Queer, Buenos Aires, Argentina: Caja Negra.

Preciado, P. B. (2002). ¿Qué es la contra-sexualidad?, Manifiesto contrasexual, Madrid, España: Opera Prima.

Rueda, A. (2019). Aportes del movimiento travesti - trans a las políticas decoloniales en América del Sur. En M. López, Los mil pequeños sexos. Intervenciones críticas sobre políticas de género y sexualidades, Sáenz Peña, Argentina: EDUNTREF. 
Vallejos, S. (2 de noviembre de 2018). DNI sin indicación de sexo y como un trámite. Página 12. Recuperado de https://www.pagina12.com.ar/152857-dni-sinindicacion-de-sexo-y-como-un-tramite

Wayar, M. (11 de mayo de 2012). ¿Qué pasó con la T?. Página 12. Recuperado de https://www.pagina12.com.ar/diario/suplementos/soy/1-2436-2012-05-11.html

\section{Cómo referenciar este artículo/How to reference this article:}

Grinstein, V. (2022). ¿El futuro es generado? Aportes para pensar en una sociedad sin encasillamientos de sexo/género. iQUAL. Revista de Género e Igualdad, 5, 116-126, doi: 10.6018/iqual.467071

Grinstein, V. (2022). ¿El futuro es generado? Aportes para pensar en una sociedad sin encasillamientos de sexo/género. [Will the future be gender-less? Key notes to reflect about a society without sex and gender stereotypes]. iQUAL. Revista de Género e lgualdad, 5, 116-126, doi: 10.6018/iqual.467071 